\title{
Application of POSSUM and P-POSSUM in Surgical Risk Assessment of Elderly Patients Undergoing Hepatobiliary and Pancreatic Surgery
}

This article was published in the following Dove Press journal: Clinical Interventions in Aging

\author{
Zhi-Wei Hu (D) \\ Rui-Qiang Xin (D) \\ Yi-Jun Xia (iD \\ Guang-Peng Jia (D) \\ Xiao-Xu Chen (ID) \\ Shi Wang (D)
}

Department of Hepatobiliary and Pancreatic Surgery, Inner Mongolia People's Hospital, Hohhot, Inner Mongolia Autonomous Region, People's Republic of China
Correspondence: Shi Wang

Department of Hepatobiliary and

Pancreatic Surgery, Inner Mongolia

People's Hospital, No. 20 Zhaowuda

Road, Hohhot, Inner Mongolia

Autonomous Region, People's Republic of

China

Tel $+8618047 \mid 92834$

$\mathrm{Fax}+864713283753$

Email18047192834@I63.com
Purpose: To investigate the efficacy and accuracy of the Physiological and Operative Severity Score for the enUmeration of Mortality and morbidity (POSSUM) and PortsmouthPOSSUM (P-POSSUM) scoring systems in the risk assessment of postoperative complications and death in elderly patients undergoing hepatobiliary and pancreatic surgery.

Patients and Methods: Using POSSUM and P-POSSUM, 274 elderly patients undergoing hepatobiliary and pancreatic surgery were evaluated, and the complications and deaths predicted by the systems were compared with the actual situation. The accuracy and predictive ability of POSSUM and P-POSSUM were evaluated using chi-squared and $t$-tests, consistency of predicted and actual complication rates (observed/expected, OE ratio), and receiver operating characteristic (ROC) curve.

Results: The complication rate predicted by POSSUM (R1) was $22.57 \%$, while the actual postoperative complication rate was $17.88 \%(\mathrm{P}>0.05)$. The mortality rate predicted by POSSUM (R2) was $4.61 \%$, while the actual rate was $1.09 \%(\mathrm{P}<0.05)$. The mortality rate predicted by P-POSSUM (R) was $1.42 \%$, while the actual rate was $1.09 \%(\mathrm{P}>0.05)$. Patients with complications had higher physiology scores (PS), operative severity scores (OS), and POSSUM scores than those without complications $(\mathrm{P}<0.05)$. Furthermore, PS, OS, and POSSUM scores were higher in the mortality group than in the survival group. However, the number of individuals in the mortality group was too small to accurately reflect the overall situation. Stratified analysis showed that consistency of the OE ratio in different subgroups was close to 1 . The ROC curve showed that the area under the curve for the complication rate predicted by POSSUM was 0.76 .

Conclusion: Although the postoperative mortality rate was higher than the actual value, POSSUM could accurately predict the postoperative complication rate in elderly patients undergoing hepatobiliary and pancreatic surgery. The P-POSSUM accurately predicted the postoperative mortality rate in this population. Patients with complications had higher POSSUM scores.

Keywords: POSSUM, P-POSSUM, elderly patients, postoperative complication rate, mortality rate

\section{Introduction}

According to the World Health Organization's reports, the world's population is aging. ${ }^{1}$

Moreover, China's aging population is also growing in number. The proportion of elderly patients undergoing surgery has also gradually increased. Various factors such as aging, multiple chronic conditions, and generalized weakness, make the 
risks of postoperative complications and death among elderly patients significantly higher than those among younger patients. Particularly in cases of hepatobiliary and pancreatic surgery, the trauma is generally significant, and there tends to be more postoperative complications. As the number of elderly patients undergoing hepatobiliary and pancreatic surgery increases every year, surgeons must address whether the elderly indeed require surgery and identify ways to reduce perioperative risk in the elderly to reduce the overall risk of postoperative complications and death. Therefore, seeking and establishing an accurate and effective preoperative assessment method is beneficial to improving the safety of surgery, reducing risks, and enhancing the quality of life among the elderly.

We used the Physiological and Operative Severity Score for the enUmeration of Mortality and morbidity (POSSUM) and Portsmouth-POSSUM (P-POSSUM) risk assessment methods to calculate the rates of postoperative complications and mortality, and postoperative mortality rate, respectively. ${ }^{2,3}$ The POSSUM scoring system was established by Copeland et al in $1991,{ }^{4}$ who used the preoperative physiological scores and intraoperative surgical scores of patients to predict the rates postoperative complications and mortality among patients, in order to assess the risk of surgery. In 1996, Whiteley et $\mathrm{al}^{5}$ found that POSSUM overestimated the postoperative mortality rate, and they revised the statistical regression equation, which was referred to as P-POSSUM. Thus far, POSSUM and P-POSSUM have been studied in colorectal and orthopedic fields, ${ }^{6,7}$ but no research has been reported in elderly patients undergoing hepatobiliary and pancreatic surgery.

This study explored the safety of surgery in 274 elderly patients admitted to our hospital for hepatobiliary and pancreatic surgery. The POSSUM and P-POSSUM scoring systems were used to predict the rates of postoperative complications and mortality. Those rates were then compared with actual conditions to determine both their clinical predictive ability and whether the two systems could provide an effective method for the surgical risk assessment of elderly patients undergoing hepatobiliary and pancreatic surgery.

\section{Patients and Methods}

\section{Clinical Information}

The clinical data of 274 elderly patients treated at the Inner Mongolia People's Hospital of Hohhot, Inner Mongolia Autonomous Region, China from October 2018 to October 2019 were considered. This study was approved by the Medical Ethics Committee of Inner Mongolia People's
Hospital (No.2018021), and its protocol was in accordance with the principles of the Declaration of Helsinki.

The inclusion criteria were as follows: elderly patients aged $\geq 60$ years who underwent surgical treatment for hepatobiliary and pancreatic diseases; provided informed consent for this study; and agreed to attend follow-up evaluation 30 days after the surgery. The exclusion criteria were: (i) patients in poor physical condition, who could not tolerate the surgery; (ii) patients who did not agree to participate in the study; and (iii) interrupted postoperative follow-up owing to various reasons, resulting in the inability to determine postoperative complications and patients mortality within 30 days of the operation. All included patients and/or their families provided written informed consent.

\section{POSSUM and P-POSSUM}

The POSSUM included 12 physiology scores (PS) and six operative severity scores (OS). Each variable had a 4-level classification, with increasing scores $(1,2,4,8)$. In the absence of data, the assigned score was $1 .^{4,8}$ Specific scoring rules are shown in Table 1. The preoperative physiological indicators used the data closest to the time of surgery. The elective surgical indicators were collected within $24 \mathrm{~h}$ before the operation, and the emergency surgical indicators were collected within $6 \mathrm{~h}$ before. The intraoperative indicators were based on the surgical and anesthesia records. Data were entered into the clinical database by another group of non-medical professionals. By substituting PS and OS into regression equations, the POSSUM scoring system predicted the postoperative complication rate (R1) and mortality rate (R2), and the P-POSSUM predicted the postoperative mortality rate (R). The calculation formula ${ }^{4,5}$ was as follows:

$$
\begin{gathered}
\ln \mathrm{R} 1 /(1-\mathrm{R} 1)=-5.91+0.16 \times \mathrm{PS}+0.19 \times \mathrm{OS} \\
\ln 2 /(1-\mathrm{R} 2)=-7.04+0.13 \times \mathrm{PS}+0.16 \times \mathrm{OS} \\
\ln \mathrm{R} /(1-\mathrm{R})=-9.065+0.1692 \times \mathrm{PS}+0.1550 \times \mathrm{OS}
\end{gathered}
$$

By substituting the PS and OS of each patient into the different regression equations above, we obtained the complication risk coefficient and mortality risk coefficient predicted by POSSUM or P-POSSUM, and then converted the risk coefficient into a percentage. Using the predicted complication rate and mortality rate of each patient, we were able to derive the overall predicted complication rate and mortality rate of all 274 patients. 
Table I Parameters Used to Calculate the POSSUM Score

\begin{tabular}{|c|c|c|c|c|}
\hline \multirow[t]{2}{*}{ Parameters } & \multicolumn{4}{|c|}{ Physiological Score } \\
\hline & $\mathbf{I}$ & 2 & 4 & 8 \\
\hline Age (years) & $\leq 60$ & $6 I-70$ & $\geq 71$ & \\
\hline Cardiac signs & $\begin{array}{l}\text { No } \\
\text { failure }\end{array}$ & $\begin{array}{l}\text { Diuretic, digoxin, antianginal, or } \\
\text { hypertensive therapy }\end{array}$ & $\begin{array}{l}\text { Peripheral edema, warfarin therapy, or } \\
\text { borderline cardiomegaly }\end{array}$ & $\begin{array}{l}\text { Raised jugular venous } \\
\text { pressure or cardiomegaly }\end{array}$ \\
\hline Respiratory signs & $\begin{array}{l}\text { No } \\
\text { dyspnea }\end{array}$ & $\begin{array}{l}\text { Dyspnea on exertion, mild } \\
\text { chronic obstructive airway } \\
\text { disease }\end{array}$ & $\begin{array}{l}\text { Limiting dyspnea (one flight), moderate } \\
\text { chronic obstructive airway disease }\end{array}$ & $\begin{array}{l}\text { Dyspnea at rest (rate } \geq 30 / \\
\text { min), fibrosis or consolidation }\end{array}$ \\
\hline $\begin{array}{l}\text { Systolic blood } \\
\text { pressure }(\mathrm{mmHg})\end{array}$ & $110-130$ & $\begin{array}{l}131-170 \\
100-109\end{array}$ & $\begin{array}{l}\geq|7| \\
90-99\end{array}$ & $\leq 89$ \\
\hline Pulse (beats/min) & $50-80$ & $\begin{array}{l}81-100 \\
40-49\end{array}$ & $101-120$ & $\begin{array}{l}\geq 121 \\
\leq 39\end{array}$ \\
\hline Glasgow coma score & 15 & $12-14$ & $9-11$ & $\leq 8$ \\
\hline Hemoglobin (g/dL) & $13-16$ & $\begin{array}{l}11.5-12.9 \\
16.1-17.0\end{array}$ & $\begin{array}{l}10.0-11.4 \\
17.1-18.0\end{array}$ & $\begin{array}{l}\leq 9.9 \\
\geq 18.1\end{array}$ \\
\hline $\begin{array}{l}\text { White cell count } \\
\left(10^{9} / \mathrm{L}\right)\end{array}$ & $4-10$ & $\begin{array}{l}10.1-20.0 \\
3.1-4.0\end{array}$ & $\begin{array}{l}\geq 20.1 \\
\leq 3.0\end{array}$ & \\
\hline Urea (mmol/L) & $\leq 7.5$ & $7.6-10.0$ & $10.1-15.0$ & $\geq 15 . \mid$ \\
\hline Sodium (mmol/L) & $\geq 136$ & $131-135$ & $126-130$ & $\leq 125$ \\
\hline Potassium (mmol/L) & $3.5-5.0$ & $\begin{array}{l}3.2-3.4 \\
5.1-5.3\end{array}$ & $\begin{array}{l}2.9-3.1 \\
5.4-5.9\end{array}$ & $\begin{array}{l}\leq 2.8 \\
\geq 6.0\end{array}$ \\
\hline Electrocardiogram & Normal & & Atrial fibrillation (rate 60-90) & Any other abnormal rhythm \\
\hline & & & & $\begin{array}{l}\text { or } \geq 5 \text { ectopics } / \mathrm{min} \text { or } \\
\mathrm{Q} \text { waves or } \mathrm{ST} / \mathrm{T} \text { wave } \\
\text { changes }\end{array}$ \\
\hline \multicolumn{5}{|c|}{ Operative severity score } \\
\hline Operative severity & Minor & Moderate & Major & Major+ \\
\hline Multiple procedures & I & & 2 & $>2$ \\
\hline Total blood loss (mL) & $\leq 100$ & $10 \mid-500$ & $501-999$ & $\geq 1000$ \\
\hline Peritoneal soiling & None & Minor (serous fluid) & Local pus & $\begin{array}{l}\text { Free bowel contest, pus, or } \\
\text { blood }\end{array}$ \\
\hline Malignancy & None & Primary only & Nodal metastasis & Distant metastasis \\
\hline Mode of surgery & Elective & & $\begin{array}{l}\text { Emergency resuscitation of }>2 \\
\mathrm{~h} \text { possible, operation }<24 \mathrm{~h} \text { after } \\
\text { admission }\end{array}$ & $\begin{array}{l}\text { Emergency (immediate } \\
\text { surgery }<2 \text { h needed) }\end{array}$ \\
\hline
\end{tabular}

Abbreviation: POSSUM, physiological and operative severity score for the enumeration of mortality and morbidity.

\section{Observation Indicators}

The actual postoperative complications and mortality data of patients were recorded, and these were divided into the complication group, mortality group, non-complication group, and survival group. Complications were defined as any event occurring within 30 days of surgery that required 
Table 2 Comparison of Predicted and Actual Values of POSSUM and P-POSSUM

\begin{tabular}{|l|l|l|l|l|}
\hline Group & Complication Rate (\%) & No. of Complications & Mortality (\%) & No. of Deaths \\
\hline POSSUM & 22.57 & 62 & $4.61 *$ & 13 \\
P-POSSUM & - & - & 1.42 & 4 \\
Actual value & 17.88 & 49 & 1.09 & 3 \\
\hline
\end{tabular}

Note: *Indicates $\mathrm{P}<0.05$ compared with actual value.

Abbreviations: POSSUM, physiological and operative severity score for the enumeration of mortality and morbidity; P-POSSUM, PortsmouthPOSSUM.

treatment not routinely applied during the postoperative period. Patients who died within 30 days postoperatively were recorded as dead. The postoperative complications and mortality data were entered into a database and compared with the POSSUM and P-POSSUM data.

The specific observation indicators were as follows: (i) general condition of patients; (ii) complication rate and mortality rate predicted by the scores; (iii) actual types of complications, complication rate, and mortality rate; (iv) comparisons of PS, OS, and POSSUM data between the complication and non-complication groups; (v) comparisons of PS, OS, POSSUM data between the mortality and survival groups; (vi) stratified analysis comparisons of the coincidence degree (observed/expected, OE ratio) between the predicted and actual complication rates in different subgroups; and (vii) receiver operating characteristic (ROC) curve, which was used to further evaluate the accuracy of POSSUM in predicting complication rate.

\section{Statistical Analysis}

Statistical analysis was performed using the SPSS 20.0 software (IBM,USA). Measurement data were expressed as mean \pm standard deviation (SD), and the non-parametric independent sample $t$-test was used for comparison. The chi-squared test was used for comparisons of countable data. The coincidence degree (OE ratio) was compared between the predicted and actual complication rates in different subgroups. The ROC curve was plotted. $\mathrm{P}<0.05$ was considered statistically significant.

\section{Results}

\section{General Condition of Patients}

The mean age of the 274 patients (115 male and 159 female) was $67.77 \pm 5.05$ years (range: $60-82$ years). The following clinical conditions were diagnosed: chronic calculous cholecystitis ( $\mathrm{n}=107)$; acute calculous cholecystitis $(\mathrm{n}=67)$; gallbladder perforation $(\mathrm{n}=6)$; common bile duct stones with cholecystitis ( $\mathrm{n}=48)$; acute obstructive suppurative cholangitis $(\mathrm{n}=19)$; hepatic cysts $(\mathrm{n}=2)$; hepatic hemangioma $(\mathrm{n}=3)$; liver abscess $(n=2)$; hepatocellular carcinoma $(n=4)$; gallbladder carcinoma $(\mathrm{n}=3)$; bile duct carcinoma $(\mathrm{n}=6)$; pancreatic cancer $(\mathrm{n}=4)$; traumatic liver rupture $(\mathrm{n}=2)$; and traumatic spleen rupture $(\mathrm{n}=1)$.

\section{Comparison of Predicted and Actual Values of POSSUM and P-POSSUM}

The POSSUM-predicted complication rate and actual rate were $22.57 \%$ and $17.88 \%$, respectively $(\mathrm{P}=0.1670$, $\left.\chi^{2}=1.909\right)$. The POSSUM-predicted mortality rate and actual rate were $4.61 \%$ and $1.09 \%$, respectively $\left(\mathrm{P}=0.0112, \chi^{2}=6.438\right)$. The P-POSSUM-predicted mortality rate was $1.42 \%\left(\mathrm{P}=0.7036, \chi^{2}=0.1447\right)$ (Table 2$)$.

\section{Types and Incidence of Complications}

Postoperative complications were classified, the number of cases was counted, and the incidence was calculated. The results are shown in Table 3.

\section{Comparison of the Complication and Non-Complication Groups}

The PS score in the complication group was higher than that in the non-complication group ( $\mathrm{P}=0.0003, t=3.872)$. The OS score of the complication group was higher than that of the non-complication group ( $\mathrm{P}<0.0001, t=5.733)$. The POSSUM score of the complication group was also higher than that of the non-complication group $(\mathrm{P}<0.0001, t=5.060)$ (Table 4).

\section{Comparison of the Mortality and Survival Groups}

The PS, OS, and POSSUM scores in the mortality group were higher than those in the survival group. However, because there were only three deaths, the size of the mortality group was too small to accurately reflect the overall situation (Table 5). 
Table 3 Types, Number, and Incidence of Complications

\begin{tabular}{|l|l|l|}
\hline Types & $\begin{array}{l}\text { No. of } \\
\text { Cases }\end{array}$ & $\begin{array}{l}\text { Complication Rate } \\
\text { (\%) }\end{array}$ \\
\hline Intestinal obstruction & 2 & 0.73 \\
\hline $\begin{array}{l}\text { Pancreatic fistula with } \\
\text { bleeding }\end{array}$ & I & 0.37 \\
\hline Pancreatic fistula & 1 & 0.37 \\
\hline Arrhythmia & 2 & 0.73 \\
\hline Pleural effusion & 1 & 0.37 \\
\hline Postoperative bleeding & 3 & 1.09 \\
\hline Acute renal impairment & 2 & 0.73 \\
Deep venous thrombosis & 3 & 1.09 \\
\hline Incision infection & 5 & 1.82 \\
\hline Cerebral infarction & 2 & 0.73 \\
\hline Urinary tract infection & 5 & 1.82 \\
\hline Septic shock & 1 & 0.37 \\
\hline Liver abscess & 1 & 0.37 \\
\hline Liver dysfunction & 5 & 1.82 \\
\hline Peritoneal effusion & 2 & 0.73 \\
\hline Celiac infection & 2 & 0.73 \\
\hline Pulmonary embolism & 1 & 0.37 \\
\hline Lung infection & 5 & 1.82 \\
\hline Biliary fistula & 5 & 1.82 \\
\hline Total & I9 & (7.88 \\
\hline
\end{tabular}

\section{Stratified Analysis of the Predicted Complication Rate}

The POSSUM-predicted complication rate (R1) was stratified, and the results showed that the predicted complication number and actual complication number in different subgroups were similar. The consistency of the OE ratio was close to 1 , which suggests that POSSUM was more accurate in predicting the complication rate in different subgroups (Table 6, Figures 1 and 2).

\section{ROC Curve for Predicting Complication Rate with POSSUM}

The ROC curve was used to further evaluate the accuracy of POSSUM in predicting the complication rate. The closer the value of the area under the curve (AUC) to 1, the
Table 4 Comparison of the Complication and NonComplication Groups

\begin{tabular}{|l|l|l|l|l|}
\hline Group & $\begin{array}{l}\text { No. of } \\
\text { Cases }\end{array}$ & PS & OS & POSSUM \\
\hline Complication & 49 & $18.55 \pm$ & $12.10 \pm$ & $30.65 \pm$ \\
group & & 4.98 & 2.64 & 6.74 \\
Non- & 225 & $15.67 \pm$ & $9.79 \pm$ & $25.46 \pm$ \\
complication & & $2.91^{*}$ & $2.03^{*}$ & $4.14^{*}$ \\
group & & & & \\
\hline
\end{tabular}

Note: *Indicates $\mathrm{P}<0.05$ compared with the complication group.

Abbreviations: PS, physiology score; OS, operative severity score; POSSUM, physiological and operative severity score for the enumeration of mortality and morbidity.

Table 5 Comparison of the Mortality and Survival Groups

\begin{tabular}{|l|l|l|l|l|}
\hline Group & $\begin{array}{l}\text { No. of } \\
\text { Cases }\end{array}$ & PS & OS & POSSUM \\
\hline Mortality & 3 & $29.33 \pm$ & $17.67 \pm$ & $47.00 \pm$ \\
group & & 7.36 & 2.49 & 5.89 \\
Survival & 271 & $16.04 \pm$ & 10.12 & $26.16 \pm$ \\
group & & 3.20 & \pm 2.18 & 4.60 \\
\hline
\end{tabular}

Abbreviations: PS, physiology score; OS, operative severity score; POSSUM, physiological and operative severity score for the enumeration of mortality and morbidity.

better the predictive effects. The results showed that the AUC was 0.76, indicating that the POSSUM was accurate, to an extent, in predicting the complication rate (Figure 3).

\section{Discussion}

Elderly patients are a high-risk group for several diseases and comprise a significant proportion of the patients requiring surgical treatment. Surgeons should accurately evaluate the risk of surgery according to the specific physical condition of each elderly patient, to determine the best time for surgery. The ideal surgical scoring system should be simple, reproducible, objective, and available to all patients, and scoring should be based on preoperative risk factors rather than intraoperative and postoperative data. ${ }^{9}$ The main goal of scoring should be to classify the patient's risk before surgery and determine the best specific treatment for the patient. However, there is still no ideal scoring system that can reliably predict surgical risk for the elderly. ${ }^{10,11}$

Many scoring systems can predict the postoperative mortality rate of patients, but few can predict the incidence of complications. The application of POSSUM has more advantages than other scoring systems because it can predict the incidence of complications while predicting the mortality rate. Because some researchers have found that POSSUM overestimates the mortality rate, Whitley et al 
Table 6 Stratified Analysis of Predicted Complication Rate

\begin{tabular}{|c|c|c|c|c|c|}
\hline RI (\%) & $\begin{array}{l}\text { No. of } \\
\text { Cases }\end{array}$ & $\begin{array}{l}\text { Average Complication } \\
\text { Rate }\end{array}$ & $\begin{array}{l}\text { Predicted Number of } \\
\text { Complications }\end{array}$ & $\begin{array}{l}\text { Actual Number of } \\
\text { Complications }\end{array}$ & $\begin{array}{l}\mathbf{O} / \\
\mathbf{E}\end{array}$ \\
\hline $0-10$ & 40 & 0.08 & 3 & 2 & 0.67 \\
\hline II-20 & 119 & 0.14 & 17 & 14 & 0.82 \\
\hline $21-30$ & 48 & 0.26 & 12 & 8 & 0.67 \\
\hline $31-40$ & 35 & 0.34 & 12 & 9 & 0.75 \\
\hline $4 I-50$ & 17 & 0.44 & 8 & 6 & 0.75 \\
\hline $5 I-60$ & 8 & 0.56 & 4 & 5 & 1.25 \\
\hline $6 I-70$ & 2 & 0.68 & I & I & 1 \\
\hline $71-80$ & 2 & 0.77 & 2 & I & 0.5 \\
\hline $8 I-90$ & I & 0.83 & 1 & 1 & 1 \\
\hline $91-100$ & 2 & 0.95 & 2 & 2 & I \\
\hline Total & 274 & - & 62 & 49 & - \\
\hline
\end{tabular}

Abbreviations: RI, the complication rate predicted by the physiological and operative severity score for the enumeration of mortality and morbidity (POSSUM); O/E, observed/expected.

modified the mortality risk formula to establish a P-POSSUM score, which can more accurately predict the mortality rate. Although P-POSSUM does not consider all factors that may affect the results, it is still the most common risk prediction model. ${ }^{12,13}$ Both POSSUM and P-POSSUM are tools used to predict the risk of postoperative complications or death, based on the patient's physiological status and severity of the operation. However, whether POSSUM and P-POSSUM can accurately predict the risk of surgery in elderly patients without distinguishing between disease and surgery type, specifically in the field of hepatobiliary and pancreatic surgery, has not yet been adequately addressed.

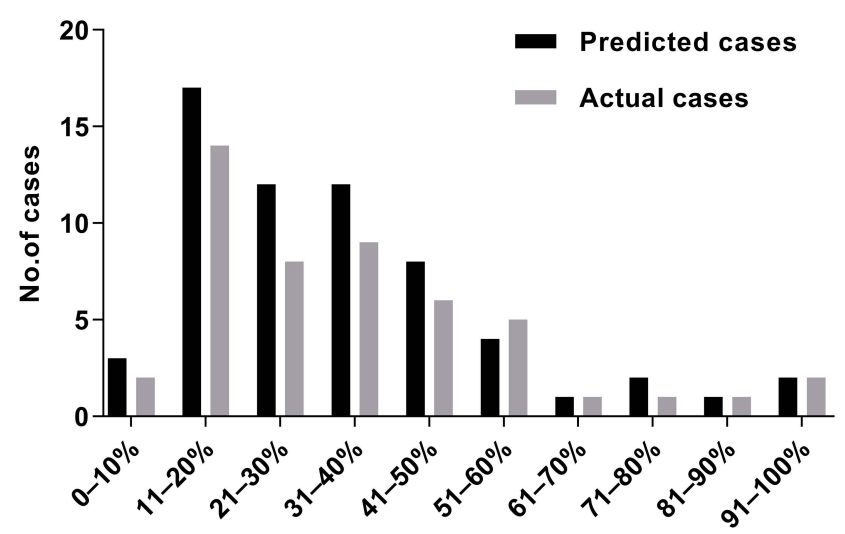

Figure I Comparison of the predicted and actual complication numbers in different subgroups.
This study proves that POSSUM can accurately predict the incidence of postoperative complications in elderly patients subjected to hepatobiliary and pancreatic surgery, but it overestimates the mortality rate of patients. These results are consistent with those of Bagnall et al. ${ }^{14}$ Furthermore, this study proves that P-POSSUM can accurately predict the postoperative mortality rate. These results are consistent with the research of Nag et $\mathrm{al}^{15}$ and Carvalho-E-Carvalho et al, ${ }^{16}$ who indicated that P-POSSUM can be used as a tool to predict postoperative mortality rate.

Further, in this study, the PS, OS, and POSSUM scores and R1 of patients with complications were compared with

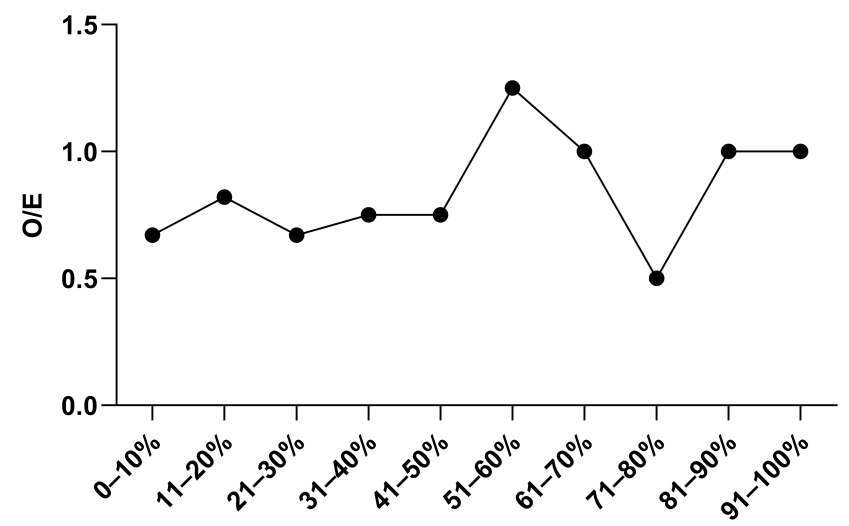

Figure 2 Comparison of the O/E in different subgroups. Abbreviation: O/E, observed/expected. 


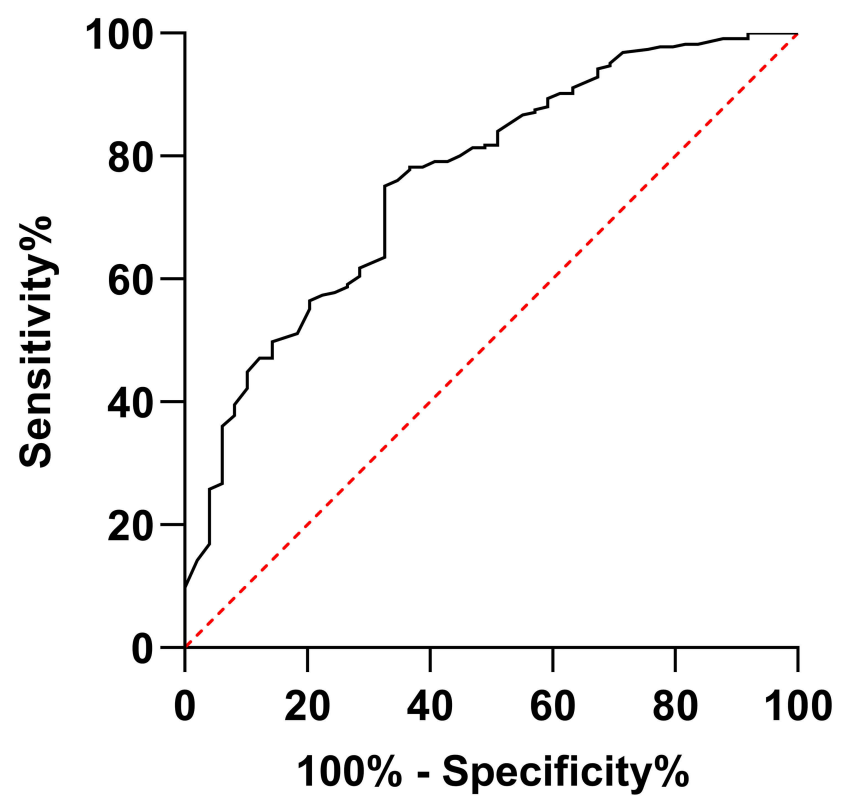

Figure 3 ROC curve to predict complication rate with POSSUM. Abbreviations: ROC, receiver operating characteristic; POSSUM, physiological and operative severity score for the enumeration of mortality and morbidity.

those of patients without complications. The results showed that patients with complications had higher PS, OS, and POSSUM scores, along with a high R1. These differences were statistically significant, which highlights the accuracy of POSSUM in predicting the complication rate from another angle. In the same way, the mortality group and survival group were compared. While the OS, POSSUM, $\mathrm{R} 2$, and R of the mortality group were significantly higher than those of the survival group, no significant intergroup differences were observed with respect to the PS. The underlying reason may be that the number of deaths was relatively small, and the differences in sample size between the two groups led to differences in the results.

To further evaluate the accuracy of POSSUM in predicting the complication rate, this study hierarchically analyzed the coincidence degree (OE ratio) between the predicted complication rate and actual complication rate in different subgroups. The $\mathrm{OE}$ ratio is the ratio of the actual value to the predicted value and mainly reflects the predictive ability of the prediction scoring system. The closer the OE ratio is to 1 , the better the prediction efficacy.

Stratified analysis showed that while the OE ratios in different subgroups differed, all were close to 1, indicating that POSSUM could accurately predict the complication rate. We also calculated the area under the ROC curve. When $0.5<\mathrm{AUC}<0.7$, the accuracy was lower; when $0.7 \leq \mathrm{AUC}<0.9$, the accuracy was somewhat improved; and when the AUC $\geq 0.9$, the accuracy was higher. Thus, POSSUM showed some accuracy in predicting the complication rate. These results indicate that POSSUM can be used as a tool to predict the rate of postoperative complications in elderly patients undergoing hepatobiliary and pancreatic surgery. This is consistent with the findings of other studies. ${ }^{17-19}$

Our study has some limitations. First, the number of deaths in this study was very small, which imposed certain limitations on data evaluation. Increasing the sample size and conducting big data research may better reflect the accuracy and validity of the scores. Second, the PS score of this study will obviously change over time, especially during emergency surgery. ${ }^{20}$ Therefore, we tried to collect the physiological indicators closest to the time of surgery. Third, although all discharged patients were followed-up for at least 30 days at the outpatient clinic, there may have been patients with complications who were not discovered but were included in the study.

\section{Conclusion}

Our results reflect the simplicity, accuracy, effectiveness, and feasibility of POSSUM and P-POSSUM. The study also found that some elderly patients have generally better health than others. This indicates that age is no longer synonymous with adverse surgical results, and the safety and necessity of surgery should be analyzed based on individual score results. We recommend the routine use of POSSUM and P-POSSUM in the postoperative risk assessment of elderly patients undergoing hepatobiliary and pancreatic surgery. Such protocols could facilitate the development of individualized surgical plans and improve the quality of life of elderly patients. Although the scoring-based diagnosis is accurate to an extent and has good clinical value, a certain gap remains from the ideal scoring system. Therefore, continuous improvement of the scoring rules based on various surgical characteristics of different medical specialties is still necessary to obtain better prediction results. $^{21}$

\section{Disclosure}

Zhiwei $\mathrm{Hu}$ and Ruiqiang Xin are co first authors. The authors report no conflicts of interest in this work.

\section{References}

1. Beard JR, Officer A, de Carvalho IA, et al. The World report on ageing and health: a policy framework for healthy ageing. Lancet. 2016;387 (10033):2145-2154. doi:10.1016/S0140-6736(15)00516-4 
2. Campillo-Soto A, Flores-Pastor B, Soria-Aledo V, et al. The POSSUM scoring system: an instrument for measuring quality in surgical patients. Cir Esp. 2006;80(6):395-399. doi:10.1016/S0009739X(06)70993-9

3. Bodea R, Hajjar NA, Bartos A, Zaharie F, Graur F, Iancu C. Evaluation of P-POSSUM risk scoring system in prediction of morbidity and mortality after pancreaticoduodenectomy. Chirurgia. 2018;113(3):399-404. doi:10.21614/chirurgia.113.3.399

4. Copeland GP, Jones D, Walters M. POSSUM: a scoring system for surgical audit. Br J Surg. 1991;78:356-360. doi:10.1002/bjs.1800780327

5. Whiteley MS, Prytherch DR, Higgins B, Weaver PC, Prout WG. An evaluation of the POSSUM surgical scoring system. Br J Surg. 1996;83(6):812-815. doi:10.1002/bjs.1800830628

6. Pinho S, Lagarto F, Gomes B, Costa L, Nunes CS, Oliveira C. CRPOSSUM and surgical Apgar score as predictive factors for patients' allocation after colorectal surgery. Rev Bras Anestesiol. 2018;68 (4):351-357. doi:10.1016/j.bjan.2018.01.002

7. Karres J, Heesakkers NA, Ultee JM, Vrouenraets BC. Predicting 30-day mortality following hip fracture surgery: evaluation of six risk prediction models. Injury. 2015;46(2):371-377. doi:10.1016/j. injury.2014.11.004

8. Lima DF, Cristelo D, Reis P, Abelha F, Mourao J. Outcome prediction with physiological and operative severity score for the enumeration of mortality and morbidity score system in elderly patients submitted to elective surgery. Saudi J Anaesth. 2019;13(1):46-51. doi:10.4103/sja.SJA_206_18

9. Barnett S, Moonesinghe SR. Clinical risk scores to guide perioperative management. Postgrad Med J. 2011;87(1030):535-541. doi:10.1136/pgmj.2010.107169

10. Teixeira IM, Teles AR, Castro JM, Azevedo LF, Mourao JB. Physiological and operative severity score for the enumeration of mortality and morbidity (POSSUM) system for outcome prediction in elderly patients undergoing major vascular surgery. J Cardiothorac Vasc Anesth. 2018;32(2):960-967. doi:10.1053/j.jvca.2017.08.036

11. Sharrock AE, McLachlan J, Chambers R, Bailey IS, Kirkby-Bott J. Emergency abdominal surgery in the elderly: can we predict mortality? World J Surg. 2017;41(2):402-409. doi:10.1007/s00268-016-3751-3

12. Simpson G, Parker A, Hopley P, Wilson J, Magee C. Pre-operative psoas major measurement compared to P-POSSUM as a prognostic indicator in over-80s undergoing emergency laparotomy. Eur J Trauma Emerg Surg. 2020;46(1):215-220. doi:10.1007/s00068-018-1025-5
13. McIlveen EC, Wright E, Shaw M, et al. A prospective cohort study characterising patients declined emergency laparotomy: survival in the 'NoLap' population. Anaesthesia. 2020;75(1):54-62. doi:10.1111/ anae. 14839

14. Bagnall NM, Pring ET, Malietzis G, et al. Perioperative risk prediction in the era of enhanced recovery: a comparison of POSSUM, ACPGBI, and E-PASS scoring systems in major surgical procedures of the colorectal surgeon. Int $J$ Colorectal Dis. 2018;33 (11):1627-1634. doi:10.1007/s00384-018-3141-4

15. Nag DS, Dembla A, Mahanty PR, et al. Comparative analysis of APACHE-II and P-POSSUM scoring systems in predicting postoperative mortality in patients undergoing emergency laparotomy. World J Clin Cases. 2019;7(16):2227-2237. doi:10.12998/wjcc.v7.i16.2227

16. Carvalho-E-Carvalho ME, De-Queiroz FL, Martins-Da-Costa BX, Werneck-Cortes MG, Pires-Rodrigues V. The applicability of POSSUM and P-POSSUM scores as predictors of morbidity and mortality in colorectal surgery. Rev Col Bras Cir. 2018;45(1):e1347. doi:10.1590/0100-6991e-20181347

17. Moonesinghe SR, Mythen MG, Das P, Rowan KM, Grocott MP. Risk stratification tools for predicting morbidity and mortality in adult patients undergoing major surgery: qualitative systematic review. Anesthesiology. 2013;119(4):959-981. doi:10.1097/ALN.0b013e3182a4e94d

18. Chatterjee AS, Renganathan DN. POSSUM: a scoring system for perforative peritonitis. J Clin Diagn Res. 2015;9(4):PC05-PC09. doi:10.7860/JCDR/2015/12720.5854

19. Ngulube A, Muguti GI, Muguti EG. Validation of POSSUM, P-POSSUM and the surgical risk scale in major general surgical operations in Harare: a prospective observational study. Ann Med Surg (Lond). 2019;41:33-39. doi:10.1016/j.amsu.2019.03.007

20. de Castro SM, Houwert JT, Lagarde SM, et al. Evaluation of POSSUM for patients undergoing pancreatoduodenectomy. World J Surg. 2009;33(7):1481-1487. doi:10.1007/s00268-009-0037-z

21. Kenig J, Mitus JW, Rapacz K, Skorus U, Pietrzyk P, Sega A. Usefulness of scoring systems in outcome prediction for older cancer patients undergoing abdominal surgery. Acta Chir Belg. 2019;1-7. doi:10.1080/00015458.2019.1642577
Clinical Interventions in Aging

\section{Publish your work in this journal}

Clinical Interventions in Aging is an international, peer-reviewed journal focusing on evidence-based reports on the value or lack thereof of treatments intended to prevent or delay the onset of maladaptive correlates of aging in human beings. This journal is indexed on PubMed Central, MedLine, CAS, Scopus and the Elsevier

\section{Dovepress}

Bibliographic databases. The manuscript management system is completely online and includes a very quick and fair peer-review system, which is all easy to use. Visit http://www.dovepress.com testimonials.php to read real quotes from published authors. 